\title{
Kryptorchismus
}

\section{Die Behandlung sollte im zweiten Lebensjahr abgeschlossen sein}

\begin{abstract}
Wird ein Kryptorchismus vor dem 13. Lebensjahr korrigiert, halbiert dies das Risiko der Betroffenen, an Hodenkrebs zu erkranken, ergab eine schwedische Studie (MMW Nr. $38 / 2007$, S. 21). Ein Hodenhochstand sollte aber noch viel früher behandelt werden, um die Fertilität des $\mathrm{Pa}$ tienten zu erhalten, mahnt ein Leser:
\end{abstract}

- Obwohl wir bereits 1977 im Deutschen Ärzteblatt $[1,2]$ auf die Notwendigkeit der Frühbehandlung des Hodenhochstandes vor Abschluss des zweiten Lebensjahres hingewiesen haben, werden auch heute noch viele Knaben mit diesem Leiden erst viel später behandelt. Die Bildunterschrift suggeriert, dass die operative Behandlung vor dem 13. Lebensjahr erfolgen soll! Das entspricht nicht der Lehrmeinung. Durch diese Zeitangabe wird aber nur darauf hingewiesen, dass eventuell das Auftreten eines Falles von
Hodenkrebs verhindert wird. Um aber die aus einer verspätet eingesetzten Therapie resultierende Infertilität nach Möglichkeit zu verhindern, sollte die Behandlung schon im zweiten Lebensjahr abgeschlossen werden. Bei einem Verbleiben der Hoden im Abdomen (Kryptorchismus) oder im Leistenkanal kommt es bereits ab dem dritten Lebensjahr zum Untergang von Präspermatogonien, was als Vorstufe der Infertilität angesehen werden muss.

\section{Literatur beim Verfasser}

- Prof. Dr. med. Otfrid Butenandt per E-Mail

\section{Antwort von}

Prof. Dr. med. H. S. Füeßl, Haar:

Streng genommen widerspricht die Bildlegende nicht Ihrer Meinung, da das zweite Lebensjahr auch vor dem 13. Lebensjahr liegt. Die Untersuchung basiert auf Daten aus dem schwedischen Krebsregister und bezieht sich damit naturgemäß nur auf die Frage des Hodenkarzinoms. Zum Fertilitätsproblem wird keine Aussage gemacht.
- Prof. Dr. med. H. S. FüeßI, Geschäftsfüh- render Schriftleiter MMW-Fortschritte der Medizin

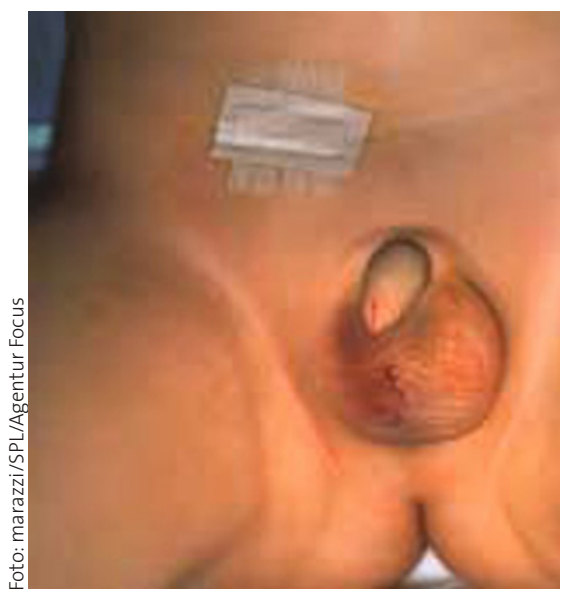

Zustand nach Orchiopexie.

\section{Warum die Lebensstiländerung bei Herzpatienten nicht klappt}

\section{Mangelnde Compliance wird auch noch belohnt}

\begin{abstract}
„Ist Ihre Bilanz auch so schlecht?", überschrieben wir unseren Bericht vom Europäischen Herzkongress in Wien in MMW Nr. 38/2007, S. 12. Wie die EuroAspire-Studien zeigen, hat es in den letzten zwölf Jahren keine Fortschritte bei den notwendigen Lebensstilkorrekturen von Herzpatienten gegeben. Dazu schreibt ein Leser:
\end{abstract}

— Durch die Überschrift „Ist Ihre Bilanz auch so schlecht?" wird impliziert, dass wir einen großen Anteil der Verantwortung für die mangelnde Compliance „unserer“ Patienten tra- gen. Dem kann ich nur widersprechen. Der aufgeklärte Patient kann von uns beraten und über die Folgen seines (meist ungesunden) Lebensstils aufgeklärt werden. Doch Fehlverhalten der Patienten wird in unserer Gesellschaft nicht sanktioniert, sondern eher finanziell motiviert: So wird der übergewichtige, rauchende und bewegungsfaule Patient durch die Einschreibung in ein DMP mit Erlassung der Praxisgebühr „belohnt".

Wäre es nicht angebracht, die Praxisgebühr dann zu erlassen, wenn realistisch umzusetzende, individuelle und messbare Ziele vereinbart und vom Pa- tienten erreicht wurden? Zum Beispiel eine Gewichtsreduktion von $5 \mathrm{~kg}$ nach sechs oder zwölf Monaten oder die Steigerung des wöchentlichen Bewegungszeitraums um 30 Minuten? Diesbezügliche Gespräche in Düsseldorf mit der BEK und AOK machten lediglich deutlich, dass die genannten Krankenkassen kein Interesse an einer so gearteten Patientenmotivation haben. Warum eigentlich nicht?

\footnotetext{
- Dr. Ralf Raßmann, Facharzt für Allgemeinmedizin, 1. Vorsitzender Hausarztnetz Düsseldorf e. V., Fliednerstraße 5, D-40489 Düsseldorf
} 